\title{
Oral metastasis of renal cell carcinoma mimicking recurrence of excised malignant myoepithelioma: A case report
}

\author{
YOSHIHIRO MORITA $^{1,2}$, TAKAKI IWAGAMI ${ }^{1,2}$, CHISATO KAWAKITA ${ }^{3}$, \\ YUKIKO KUSUYAMA $^{1,2}$, ATSUKO NIKI-YONEKAWA ${ }^{1,2}$ and NOBUO MORITA ${ }^{1,3}$ \\ ${ }^{1}$ Department of Oral and Maxillofacial Surgery, Seichokai Hannan Municipal Hospital, Hannan, \\ Osaka 599-0202; ${ }^{2}$ Department of Oral and Maxillofacial Surgery II, Osaka University Graduate \\ School of Dentistry, Suita, Osaka 565-0871; ${ }^{3}$ Department of Oral-Maxillofacial Surgery, NS Medical \\ and Healthcare Service General Incorporation Foundation, Wakayama 640-8555, Japan
}

Received September 7, 2017; Accepted May 16, 2018

DOI: $10.3892 / \mathrm{mco} .2018 .1630$

\begin{abstract}
Following breast and lung cancers, renal cell carcinoma $(\mathrm{RCC})$ is the third most frequent cancer to metastasize to the head and neck region, though such cases are rarely reported. Distinguishing between malignant tumors of salivary gland origin and metastatic RCC is very important. The case of a 75-year-old man with an oral cavity lesion in the left buccal submucosa measuring 40x30 mm that had grown substantially over several weeks is presented. His medical history included left kidney cancer 26 years earlier and a malignant myoepithelioma of the left buccal region 7 years earlier. It was suspected that this lesion was a recurrent malignant myoepithelioma as it appeared at the same site as the previous operation. Surgery was performed, and metastatic RCC was confirmed upon pathological examination. The diagnosis of metastatic RCC was made by immunohistochemical examination, which also excluded malignant myoepithelioma and other clear cell carcinomas of salivary gland origin. Metastatic RCC must be considered in the differential diagnosis of a new oral cavity lesion presenting in a patient with a past history of kidney cancer. Thus, immunohistochemical staining is required to distinguish malignancies of salivary gland origin, including malignant myoepithelioma, from metastatic RCC.
\end{abstract}

\section{Introduction}

The oral cavity is an extremely rare site for metastases, since metastases account for approximately $1 \%$ of all malignant tumors in the oral cavity. The most common primary tumors that metastasize to the oral cavity are lung carcinoma in

Correspondence to: Dr Yoshihiro Morita, Department of Oral and Maxillofacial Surgery II, Osaka University Graduate School of Dentistry, 1-8 Yamadaoka, Suita, Osaka 565-0871, Japan

E-mail: yoshisportsbaka@gmail.com

Key words: oral metastasis, metastatic renal cell carcinoma, malignant myoepithelioma, clear cell carcinoma, salivary gland males and breast carcinoma in females, followed by renal cell carcinoma (RCC) (1). Men between the ages of 30 and 60 years are the group most commonly affected by RCC (2). Metastases develop in approximately one-third of RCC cases, and approximately one-half of RCC metastases are distant metastases that are seen following the initial diagnosis. Distant metastases from RCC most commonly affect the lungs, bone, liver, adrenal glands, contralateral kidney, and brain (3).

Myoepitheliomas are rare tumors of myoepithelial differentiation, which account for $1.5 \%$ of all salivary gland tumors. Malignant myoepithelioma is much rarer; $<2 \%$ of all salivary gland carcinomas are malignant myoepitheliomas (4). Distinguishing RCC from malignancies of salivary gland origin is very important.

A case of RCC metastasis to the oral cavity that initially presented with a left buccal submucosal swelling is presented. In this patient, a malignant myoepithelioma was removed surgically from buccal submucosa at the same site in another hospital eleven years earlier.

\section{Case report}

A 75-year-old man was referred to our outpatient clinic for an oral cavity lesion involving the left buccal submucosa. The lesion had grown substantially over several weeks. His family history was unremarkable. His past history included left kidney cancer treated 26 years earlier and a malignant myoepithelioma that was removed surgically from the buccal region at the same site at another hospital eleven years earlier. He had facial asymmetry, with diffuse swelling of the left side of the cheek. On physical examination, a soft mass with a smooth surface was seen in the oral cavity involving the left side buccal mucosa, measuring 40x30 $\mathrm{mm}^{2}$. Contrastenhanced computed tomography (CECT) of the face showed a $40 \times 35 \times 35 \mathrm{~mm}^{3}$, ill-defined, soft tissue mass lesion in the left side buccal submucosa (Fig. 1A and B). On magnetic resonance imaging (MRI), there was a 40x40x35 $\mathrm{mm}^{3}$, well-circumscribed mass that showed high and nonhomogeneous signal intensity on the left side under the buccal mucous membrane (Fig. 2A and B). Blood and serum biochemistry examinations were within normal limits. Suspecting that this 


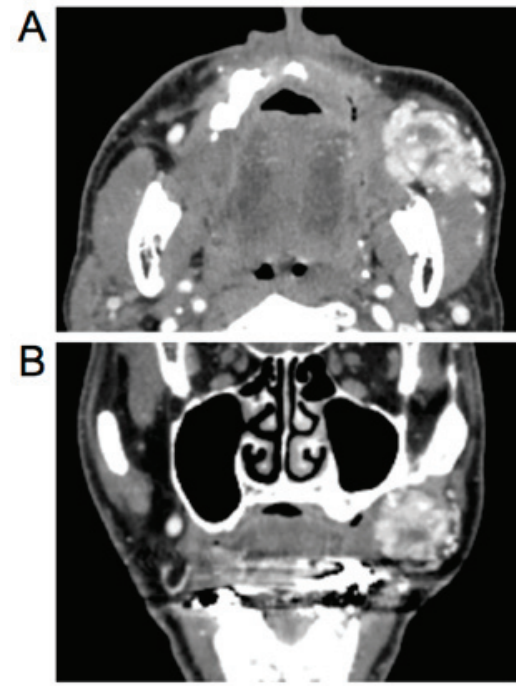

Figure 1. Contrast-enhanced computed tomography imaging. (A) Axial

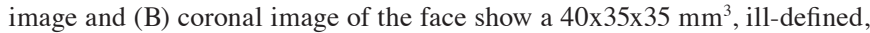
soft tissue mass lesion in the left side buccal mucosa.

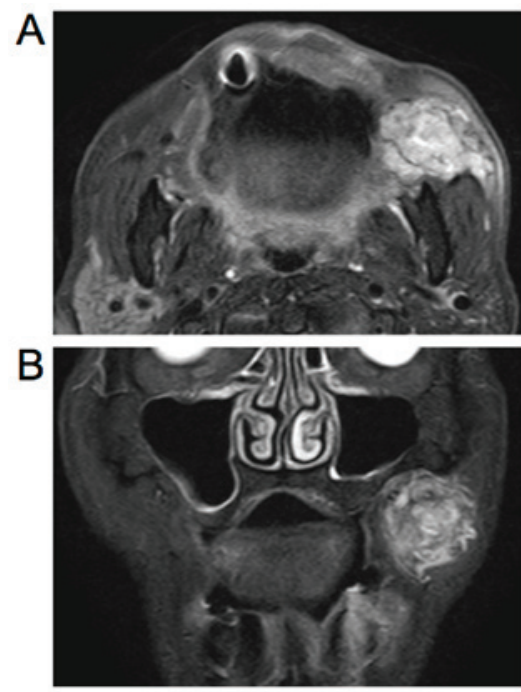

Figure 2. Magnetic resonance imaging. (A) Axial T2-weighted image and

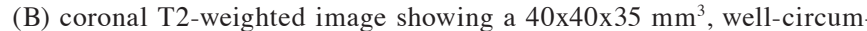
scribed mass with high and nonhomogeneous signal intensity on the left side beneath the buccal mucous membrane.

tumor was recurrent malignant myoepithelioma, surgery was performed.

The lesion was removed via an intraoral incision of the left buccal mucosa under general anesthesia. During surgery, the mass was approached by a transverse $5-\mathrm{cm}$ linear incision made in the mucous lining overlying it. The irregular mass was carefully excised with a 10 to $15-\mathrm{mm}$ safety margin (Fig. 3A and B), and the wound was closed using sutures.

On histopathologic examination, the metastatic origin of the submucous lesion was confirmed by images that were compatible with clear-cell carcinoma (Fig. 4A). The use of immunohistochemical techniques confirmed its renal origin (Fig. 4B-E). Histopathologic analysis was performed on formalin fixed paraffin embedded sections $(4 \mu \mathrm{m})$. Hematoxylin and eosin (H\&E) staining was performed at
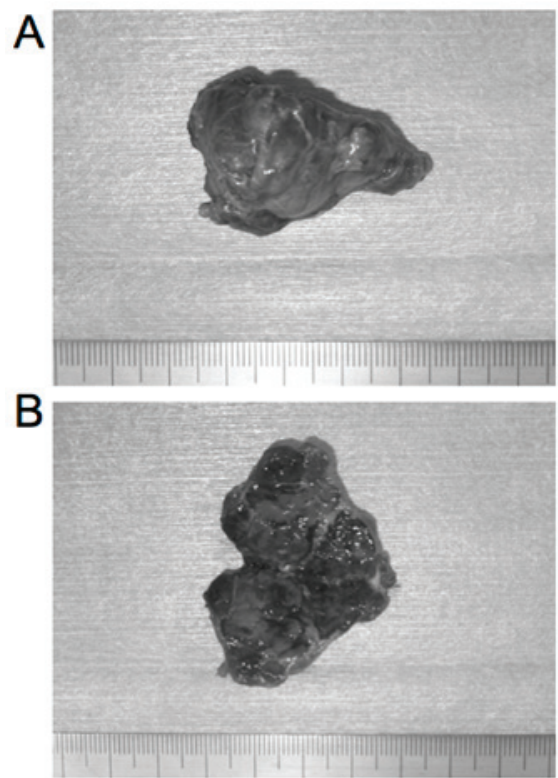

Figure 3. Surgical specimen. (A) Macroscopic view of the tumor mass following excision with a 10 to $15-\mathrm{mm}$ safety margin. (B) A cut surface of the tumor mass.

room temperature (staining in hematoxylin for $3 \mathrm{~min}$ and eosin for $2 \mathrm{~min}$ ). Immunohistochemical staining for S100 protein (cat. no. N1517; 1:3 dilution; Dako Corporation, Carpinteria, CA, USA), $\alpha$ SMA (cat. no. 712021; predilution antibody; Nichirei Biosciences Inc., Tokyo, Japan), p63 (cat. no. 713751, Nichirei biosciences Inc., predilution antibody), and CD10 (cat. no. 713261; predilution antibody; Nichirei Biosciences Inc.) was performed, and it was positive only for CD10. Therefore, the lesion was diagnosed as oral metastasis of RCC. The symptoms resolved after the operation. Postoperative follow-up at 22 months showed good healing without evidence of recurrence. The patient has given his consent for this case report to be published.

\section{Discussion}

Metastases to the oral cavity are extremely rare, and they likely occur through the arterial, venous, and lymphatic circulations. In the head and neck region, it has been reported that RCC metastasizes to the nose, tongue, paranasal sinuses, parotid glands, larynx, mandible, temporal bone, and thyroid gland (3,5). Meanwhile, malignant myeoepithelioma is also a much rarer lesion that can occur in all salivary glands. In 1975, Stromeyer described the first case of malignant myoepithelioma in the parotid $(4,6)$. This malignant disease was defined by Ellis in 1991 and appeared as a distinct clinicopathological entity for the first time in the WHO classification in the same year $(4,6)$. Since that time, there have been reports of many cases affecting the parotid gland. Patients' mean age at the time of diagnosis is 55 years (range 14-86 years), with no sex difference, and $75 \%$ of all malignant myeoepitheliomas develop in the parotid, but they are also found in the submandibular and other minor salivary glands (4). In a previous study, all cases of malignant myeoepithelioma were treated by surgical resection (6).

If we suspect a salivary gland tumor, a fine-needle aspiration biopsy is usually performed. However, the morphology and 

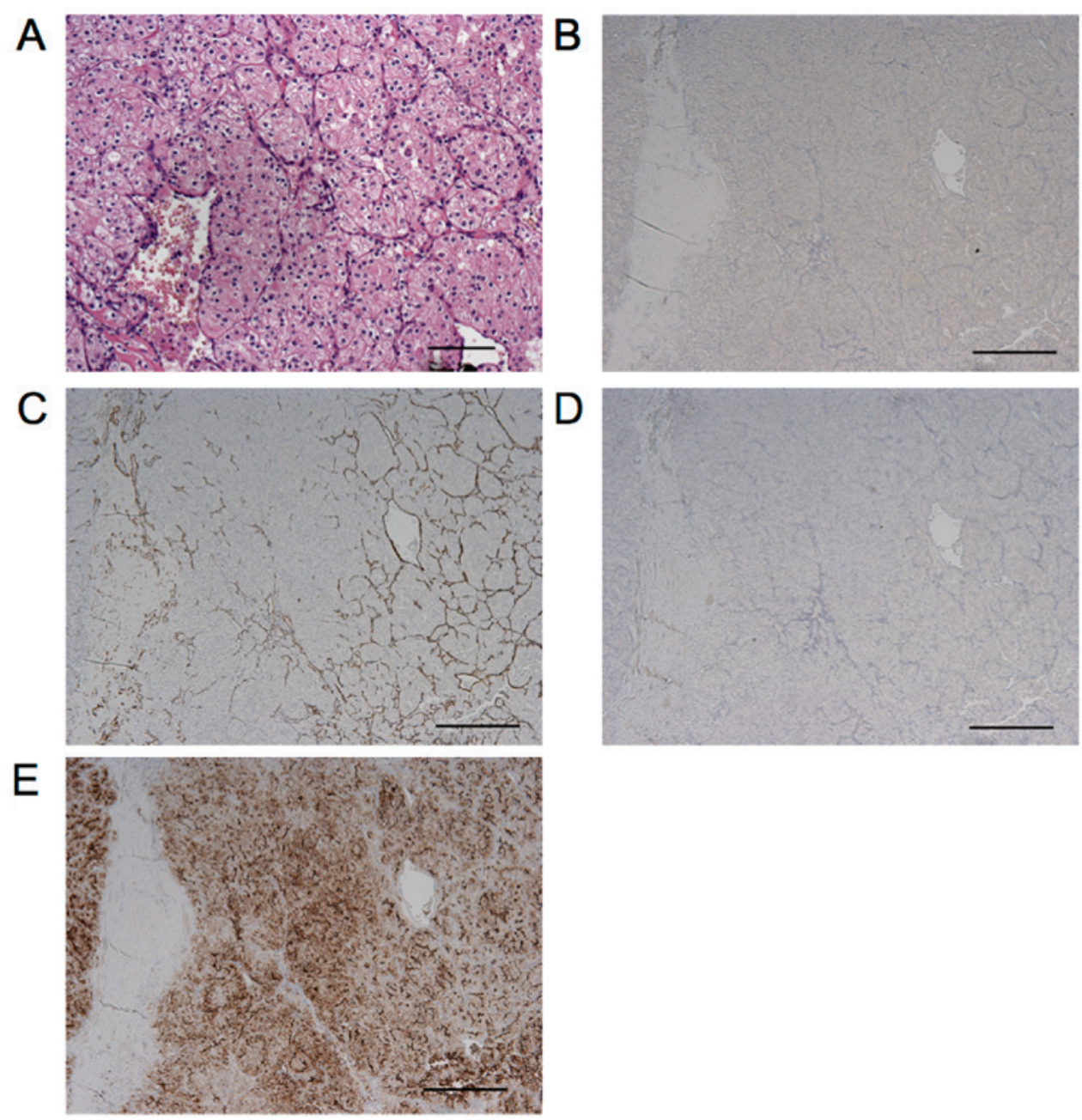

Figure 4. Photomicrograph showing (A) that the tumor growth patterns are alveolar with solid sheets of clear or acidophilic cytoplasm cells separated by hyalinized stroma (hematoxylin and eosin staining). (B) Immunohistochemistry showing that the cells were negative for S100. (C) Immunohistochemistry revealing that the cells were negative for $\alpha$-smooth muscle actin. (D) Immunohistochemistry showing that the cells were negative for p63. (E) Immunocytochemistry revealing a strong positive reaction for Cluster of Differentiation 10. Scale bars, (A) $100 \mu \mathrm{m}$ and (B-E) $500 \mu \mathrm{m}$; magnification, (A) x200 and (B-E) x50.

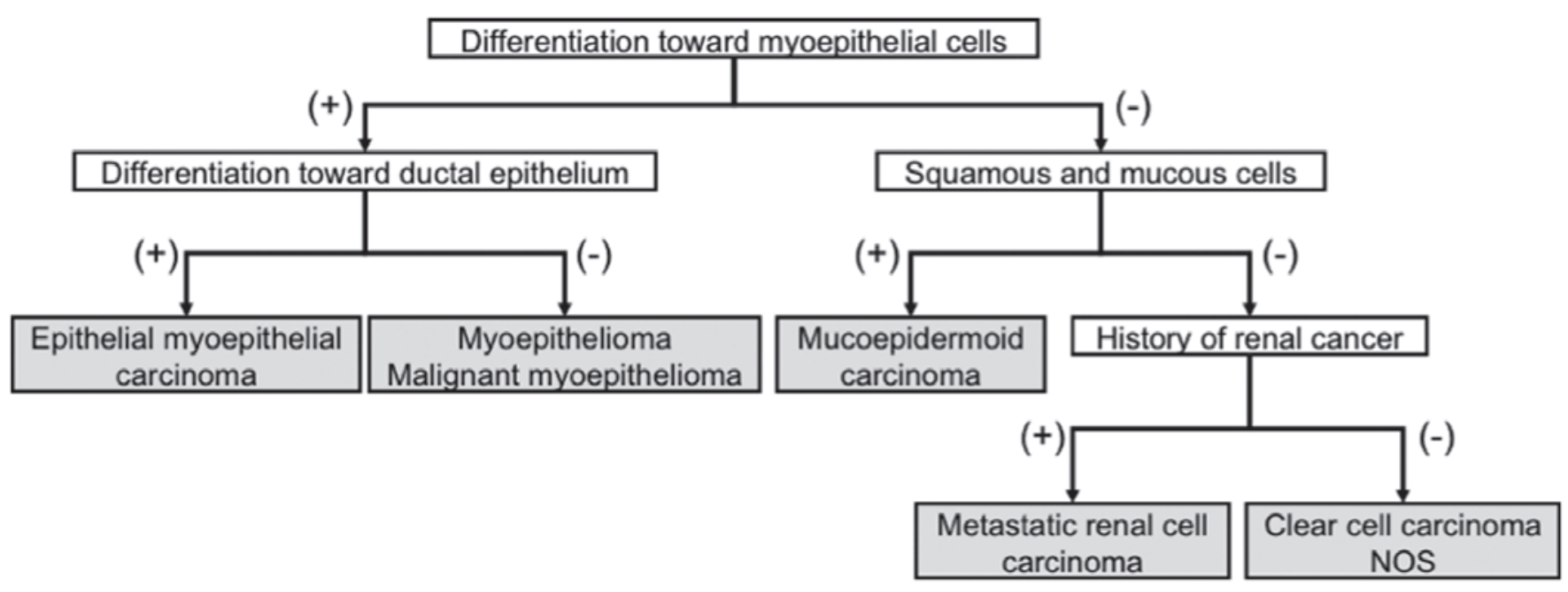

Figure 5. Schematic of the differential diagnosis of clear cell tumors in the oral cavity. NOS, nitric oxide synthase.

histology of metastatic RCC are often very similar to the primary renal lesion. There is a high risk of bleeding following fine-needle aspiration biopsy of RCC involving the kidney; up to $90 \%$ of patients show evidence of perinephric bleeding on CT, with clinically significant hemorrhage seen in 5-7\% $(3,7)$. Therefore, when a biopsy is performed for clinical suspicion 
of RCC metastasis, hemorrhage should be expected. In a previous study, suggested measures to improve the prognosis of patients with RCC included early diagnosis of metastases, nephrectomy, and metastasectomy (8). In the present case, fineneedle aspiration biopsy was not performed for several reasons. Recurrent malignant myoepithelioma was strongly suspected because the patient underwent surgery for a malignant myoepithelioma from the same site in another hospital eleven years earlier. This patient was an elderly person, 75 years old, and there was a risk of bleeding with biopsy of a metastatic RCC lesion. Whether it was a malignant myoepithelioma or metastatic RCC, tumor removal was needed. Therefore, tumor removal was performed without biopsy. After surgery, this lesion was postoperatively diagnosed histologically as metastasis of RCC.

Differentiating among clear cell tumors histologically is difficult by conventional light microscopy alone. This is especially true when trying to distinguish RCC metastases from clear cell malignancies of the salivary glands. Clear cell carcinomas of the salivary glands are usually seen as nests of clear cells divided by thin, fibrous connective septa and irregular vascular tissue. However, immunohistochemical staining can help make the diagnosis, since RCC metastases show a strong reaction to vimentin and focal cytokeratin positivity, while minor salivary gland cancers show diffuse cytokeratin positivity (9). Most malignant myoepitheliomas are usually less monomorphic than benign myoepitheliomas. They frequently have high mitotic activity and atypical forms (4). Variable expressions of vimentin, broad-spectrum cytokeratin, and other myoepithelial markers, including S100, $\alpha$ SMA, GFAP, CD10, calponin, maspin, and SMMHC (smooth muscle myosin heavy chain) have been shown in various immunohistological studies $(4,6)$. Immunohistochemical staining for p63 may be useful for distinguishing mucoepidermoid carcinoma from some clear cell tumors (10). CD10 expression in RCC may be useful as a marker in the differential diagnosis of several tumors. A chart of the differential diagnosis of clear cell tumors is shown in Fig. 5. In the present case, there was no differentiation to myoepithelial cells on immunostaining because myoepithelial markers such as S100, $\alpha$ SMA, and p63 were negative, excluding CD10. Moreover, immunohistochemically, CD10 was positive, and the patient had a past history of kidney cancer. Positive and negative controls were used for immunostaining to evaluate staining status (data not shown). Therefore, it was possible to diagnose the metastatic RCC. In addition, although it could not be clearly confirmed, the lesion that had been removed 11 years earlier might actually have also been metastasis of RCC.

RCC is known to rarely metastasize to the head and neck region. Therefore, in a patient with a history of RCC, metastatic RCC should be considered in the differential diagnosis of an oral or neck lesion. In patients with a clear cell carcinoma of the mouth, immunohistochemical staining is important to differentiate between metastatic RCC and malignant tumors of salivary gland origin.

\section{Acknowledgements}

Not applicable.

\section{Funding}

No funding was received.

\section{Availability of data and materials}

The datasets used and/or analyzed during the current study are available from the corresponding author on reasonable request.

\section{Authors' contributions}

YM and NM conceived and designed the present study. YM, TI, CK and NM acquired the data. YM, YK and ANY performed data analysis and interpreted the results. YM and TI drafted the manuscript, and YM, TI, ANY and NM critically revised the manuscript for important intellectual content. All authors gave approval for the version of the manuscript to be published.

\section{Ethics approval and consent to participate}

Not applicable.

\section{Consent for publication}

The patient provided written informed consent for the publication of their data.

\section{Competing interests}

The authors declare that they have no competing interests.

\section{References}

1. Pritchyk KM, Schiff BA, Newkirk KA, Krowiak E and Deeb ZE: Metastatic renal cell carcinoma to the head and neck. Laryngoscope 112: 1598-1602, 2002.

2. Cheng ET, Greene D and Koch RJ: Metastatic renal cell carcinoma to the nose. Otolaryngol Head Neck Surg 122: 464, 2000.

3. Will TA, Agarwal N and Petruzzelli GJ: Oral cavity metastasis of renal cell carcinoma: A case report. J Med Case Reports 2: 313, 2008.

4. Richa, Ray JG, Mohanty SP and Vibha : Richa, Ray JG, Mohanty SP and Vibha: Malignant myoepithelioma of palate. Contemp Clin Dent 3: 370-372, 2012.

5. Torres-Carranza E, Garcia-Perla A, Infante-Cossio P, Belmonte-Caro R, Loizaga-Iriondo J-M and Gutierrez-Perez J-L: Airway obstruction due to metastatic renal cell carcinoma to the tongue. Oral Surg Oral Med Oral Pathol Oral Radiol Endod 101: e76-e78, 2006.

6. Patrocinio LG, Damasceno PG and Patrocinio JA: Malignant myoepithelioma of the hard palate: 9-year follow-up. Rev Bras Otorrinolaringol (Engl Ed) 75: 620, 2009.

7. Vassiliades VG and Bernardino ME: Percutaneous renal and adrenal biopsies. Cardiovasc Intervent Radiol 14: 50-54, 1991.

8. Naito S, Yamamoto N, Takayama T, Muramoto M, Shinohara N, Nishiyama K, Takahashi A, Maruyama R, Saika T, Hoshi S, et al: Prognosis of Japanese metastatic renal cell carcinoma patients in the cytokine era: A cooperative group report of 1463 patients. Eur Urol 57: 317-325, 2010.

9. Marioni G, Gaio E, Poletti A, Derosas F and Staffieri A: Uncommon metastatic site of renal adenocarcinoma: The oral tongue. Acta Otolaryngol 124: 197-201, 2004

10. O'Sullivan-Mejia ED, Massey HD, Faquin WC and Powers CN: Hyalinizing clear cell carcinoma: Report of eight cases and a review of literature. Head Neck Pathol 3: 179-185, 2009. 